\title{
Prediction of functional outcome after spinal cord injury: a task for the rehabilitation team and the patient
}

\author{
MC Schönherr*1,2,3, JW Groothoff ${ }^{2}$, GA Mulder ${ }^{1,2}$ and WH Eisma ${ }^{2,3}$ \\ ${ }^{1}$ Beatrixoord Rehabilitation Centre, Haren, The Netherlands; ${ }^{2}$ Northern Centre for Health Care Research, University \\ of Groningen, Groningen, The Netherlands; ${ }^{3}$ Department of Rehabilitation, University Hospital Groningen, Groningen, \\ The Netherlands
}

\begin{abstract}
Study design: Descriptive analysis of data gathered in an information system.
Objectives: To explore the predictions of professionals and patients regarding functional outcome after spinal cord injury related to the final results after inpatient rehabilitation, in order to make prognostics of rehabilitation outcome more successful and enlarge the role of the patient in selecting realistic rehabilitation goals.

Methods: Data from 55 patients with spinal cord injury admitted to the rehabilitation centre. Expectations of the rehabilitation team and the patients regarding future independence in performing six daily activities were compared to the functional results at discharge. The results of patients with different level and extent of lesion were analyzed.

Results: In 52\% of all performed skills, independence was achieved at discharge. Professionals and patients made similar predictions. If they both expected independence after rehabilitation, $90 \%$ of the skills were performed independently at discharge. If they both did not expect independence only $3 \%$ of the functional results were positive. Of all combined predictions $64 \%$ was correct. Correct predictions were most often found regarding self-care skills of patients with paraplegia and regarding mobility of patients with complete lesions. Prediction of self-care outcome of patients with tetraplegia is far more complicated. There was a considerable variation in predictions of mobility potential, especially regarding patients with incomplete lesions. If the team and patients agreed upon expected independence in mobility skills of these patients, the final results were mostly positive.

Conclusions: Prediction of functional outcome after spinal cord injury was most successful if the expectations of the team and patients were combined. Prognosis of self-care outcome of patients with paraplegia and mobility potential of patients with complete spinal cord lesions was usually clear at admission. However, selection of realistic goals concerning self-care skills of patients with tetraplegia and mobility skills of patients with incomplete lesions is far more complicated. Gradual adjustment of objectives is needed during the rehabilitation process in close collaboration between the professionals and the patients.
\end{abstract}

Spinal Cord (2000) 38, 185-191

Keywords: spinal cord injury; rehabilitation; functional outcome; activities of daily living; prediction

\section{Introduction}

Soon after their accident patients with spinal cord injuries (SCI) are confronted with motor and sensory deficits and bladder and bowel dysfunction of which the consequences for daily living are uncertain. Early prediction of neurological recovery and functional abilities after rehabilitation is essentially to inform the patient and to make plans for treatment. Several studies focused on neurological and functional outcome after spinal cord lesions. ${ }^{1-6}$ However, studies

*Correspondence: MC Schönherr, Beatrixoord Rehabilitation Centre, P.O. Box 30.002, 9750 RA Haren, The Netherlands that actually evaluate functional prognosis are rare $^{4}$ and expectations of patients were never examined, as far as we know. This study was conducted to explore the predictions of both the professionals and the patients regarding functional outcome, related to the final results after rehabilitation.

In the acute phase, prognosis is usually based on neurological status and early restoration of motor and sensory functions. In several studies motor and sensory recovery after SCI was quantified based on the initial neurological level of injury. ${ }^{1-3}$

To select realistic goals and involve patients in their rehabilitation process, information about the prog- 
nosis of functional outcome is indispensable., ${ }^{4,7}$ Prediction of functional potential is most realistic after 30 days, when major changes in neurological status have taken place. ${ }^{1}$ In general, we base our prognosis of functional outcome on the level and extent of lesion. Theoretical models are developed to predict potential physical abilities. ${ }^{4,5,7-9}$ Little is known about the actual number of patients who achieve this theoretical level of independence. Other factors like age, weight, co-morbidity, spasticity, coping strategies and motivation also seem to play an important role. $^{4-6,8,10,11}$ According to their physical potential most patients with paraplegia can achieve independence in self-care skills. ${ }^{4}$ There is a considerable difference in opinion regarding the patient's ability to walk independently. ${ }^{12}$

Many patients with spinal cord injuries are able to go home after the rehabilitation period and a significant number achieve a reasonable degree of independence in the performance of daily living skills. $^{4-6,13}$ Quality of life is more difficult to predict and mainly determined by effective adjustment to the changes after the injury.

We were interested to see if the expectations of professionals and patients concerning functional outcome were realistic. In this study the predictions of independence in self-care and mobility skills assessed soon after admission were related to the functional status at discharge. In order to explore the role of the patients, we compared their predictions with those of the professionals. Combinations of predictions of both the team and patients were studied. Differences in predicting outcome of patients with complete and incomplete tetraplegia and paraplegia were analyzed.

\section{Methods}

This retrospective study was based on the data of 55 patients with acute spinal cord injury who were admitted to Beatrixoord Rehabilitation Centre from 1988 to 1994 . The SCI department treats patients with acute and prolonged SCI, and patients with other severe injuries and non-traumatic diseases of the spinal cord.

Data were registered in the Rehabilitation Information System - Information System for patients with spinal cord injury (RIS-DIS). This information system was developed to evaluate the rehabilitation treatment of patients with SCI and to work out a prognostic model for functional outcome. Four members of the rehabilitation team (physician for rehabilitation medicine, nurse, physical therapist and occupational therapist) provided detailed information about the medical and functional course of patients from admission to discharge following uniform instructions.

At 8 weeks after admission the rehabilitation team was asked to predict the functional status of individual patients with acute SCI regarding several daily activities. The medical and psychosocial situation of the patient was taken into account. Questions were asked such as 'Do you expect that the patient will eat independently?' 'Do you expect that the patient will walk independently?', etc. Possible answers were 'yes', 'no' and 'uncertain'. The rehabilitation team reported 'yes' or 'no', if all members agreed upon the expectation. If a unanimous prediction was not available, 'uncertain' was recorded.

At the same time each patient also filled in this questionnaire assisted by the social worker, neither being informed about the answers of the team. The patient followed a standard rehabilitation programme from the first day after admission including education and training.

For this study the following were used (1) the predictions reported by the team and the patients 8 weeks after admission and (2) the functional results registered in the RIS-DIS assessed by the occupational therapist at discharge. Functional outcome was expressed in terms of independence in the six activities of daily living. Of all functional activities in the RISDIS we selected eating, upper body dressing and lower body dressing (self-care), walking, stair climbing and making a car transfer (mobility). Independence was defined as the observed ability to perform an activity without the help of another person (with or without appliance or orthosis). Independent walking was defined as the ability to walk about $50 \mathrm{~m}$ with or without devices. The therapist who assessed the functional results was blinded from the expectations at admission.

Data of functional status at discharge of all patients were present. Predictions of the rehabilitation team were missing in three cases, predictions of the patients in two cases and of both the rehabilitation team and patients in six cases. All 11 cases were left out of the study.

In order to discriminate for level and extent of lesion the study group was divided in to four subgroups: (1) complete tetraplegia; (2) complete paraplegia; (3) incomplete tetraplegia and (4) incomplete paraplegia. According to the standards for neurological and functional classification by the American Spinal Cord Association of 1992 the neurological level was defined as the most caudal segment of the spinal cord with normal function. ${ }^{14}$ All lesions that were complete at admission remained complete during the course.

For the analysis of age as a discriminating factor we divided the population into groups: (1) below 30 years of age, and (2) 30 years and older. We differentiated between spasticity of upper and lower extremities, spasticity of lower extremities and no spasticity at all. Data analysis was done by the SPSS/PC programme.

\section{Results}

The study group consisted of 55 patients with a spinal cord injury of which 11 cases were left out because data of predictions were missing. This group consisted of seven patients with an incomplete tetraplegia, who 
were almost fully independent and able to walk soon after admission. They were often discharged before assessment of predictions regarding future abilities took place. One patient was unable to answer the questions because of poor knowledge of Dutch.

The remaining group of 44 patients included six females and 38 males. Their age ranged from 16 to 73 years with a mean of 32 years. Half of the injuries were caused by traffic accidents. Twenty-eight patients $(64 \%)$ underwent surgical interventions and ten patients $(23 \%)$ were treated with traction or longterm immobilisation. The mean length of stay in hospital in the acute phase was 28 days. The stay in the rehabilitation centre lasted on average 270 days (range 107 to 514 days). Ten patients had complete tetraplegia, 18 patients complete paraplegia, 11 patients incomplete tetraplegia and five patients had incomplete paraplegia.

In Table 1 the predictions of the rehabilitation team are compared with those of the patients and we found that $140(53 \%)$ versus $147(56 \%)$ 'yes' answers were given and $73(28 \%)$ versus $76(29 \%)$ 'no' answers. We found $51(19 \%)$ 'uncertain' answers given by the team and $41(16 \%)$ by the patients. The team was more reserved in predicting results of self-care skills and car transfer. The patients were more uncertain about the independence in stair climbing. 'Uncertain' expectations of independence in walking were equal in both groups.

Of 140 activities in which independence was predicted by the team 119 of $140(85 \%)$ results were positive compared to 120 of $147(82 \%)$ positive results predicted by the patients. When the team and patients did not expect independence, in only four of $73(5 \%)$ respectively three of 76 activities $(4 \%)$ positive results were achieved. These differences were not significant. In 138 of all 264 performed activities (52\%) independence was achieved at discharge. Upper body dressing was performed independently in $80 \%$ of the patients and independence in eating was found in $68 \%$ of patients. One out of four patients was able to walk independently at discharge.

In Table 2 identical predictions of the rehabilitation team and patients concerning six activities of daily living were combined. Of 264 combined answers we found $123(47 \%)$ both 'yes'-combinations, meaning that both the team and the patient expected independence and $59(22 \%)$ both 'no'-combinations with unanimous negative expectations. Of the both 'yes'-combinations 111 of $123(90 \%)$ were correct, meaning that they were in accordance with the final results. Of the 59 both 'no'-combinations 57 (97\%)

Table 1 Predictions of the rehabilitation team and patients regarding independence in different activities of daily living related to number and percentage of positive results in performing activities independently at discharge $(n=44)$

\begin{tabular}{|c|c|c|c|c|c|c|c|}
\hline \multirow[b]{2}{*}{ Activities of daily living } & \multirow[b]{2}{*}{ Predictions } & \multicolumn{2}{|c|}{ Rehabilitation team } & \multirow[b]{2}{*}{$\%$} & \multicolumn{2}{|c|}{ Patients } & \multirow[b]{2}{*}{$\%$} \\
\hline & & $n$ & Pos. result & & $n$ & Pos. result & \\
\hline \multirow[t]{4}{*}{ Eating } & Yes & 32 & 26 & 81 & 34 & 28 & 82 \\
\hline & No & 2 & 0 & 0 & 3 & 1 & 33 \\
\hline & Uncertain & 10 & 4 & 40 & 7 & 1 & 14 \\
\hline & Total & 44 & 30 & 68 & 44 & 30 & 68 \\
\hline \multirow[t]{4}{*}{ Upper body dressing } & Yes & 38 & 32 & 84 & 38 & 34 & 89 \\
\hline & No & 1 & 0 & 0 & 3 & 0 & 0 \\
\hline & Uncertain & 5 & 3 & 60 & 3 & 1 & 33 \\
\hline & Total & 44 & 35 & 80 & 44 & 35 & 80 \\
\hline \multirow[t]{4}{*}{ Lower body dressing } & Yes & 26 & 23 & 88 & 28 & 21 & 75 \\
\hline & No & 3 & 0 & 0 & 6 & 0 & 0 \\
\hline & Uncertain & 15 & 4 & 27 & 10 & 6 & 60 \\
\hline & Total & 44 & 27 & 61 & 44 & 27 & 61 \\
\hline \multirow[t]{4}{*}{ Car transfer } & Yes & 30 & 26 & 87 & 32 & 25 & 78 \\
\hline & No & 3 & 0 & 0 & 5 & 0 & 0 \\
\hline & Uncertain & 11 & 2 & 18 & 7 & 3 & 43 \\
\hline & Total & 44 & 28 & 64 & 44 & 28 & 64 \\
\hline \multirow[t]{4}{*}{ Walking } & Yes & 7 & 6 & 86 & 8 & 6 & 75 \\
\hline & No & 28 & 3 & 11 & 27 & 1 & 4 \\
\hline & Uncertain & 9 & 2 & 22 & 9 & 4 & 44 \\
\hline & Total & 44 & 11 & 25 & 44 & 11 & 25 \\
\hline \multirow[t]{4}{*}{ Stair climbing } & Yes & 7 & 6 & 86 & 7 & 6 & 86 \\
\hline & No & 36 & 1 & 3 & 32 & 1 & 3 \\
\hline & Uncertain & 1 & 0 & 0 & 5 & 0 & 0 \\
\hline & Total & 44 & 7 & 16 & 44 & 7 & 16 \\
\hline \multirow[t]{4}{*}{ Total } & Yes & 140 & 119 & 85 & 147 & 120 & 82 \\
\hline & No & 73 & 4 & 5 & 76 & 3 & 4 \\
\hline & Uncertain & 51 & 15 & 29 & 41 & 15 & 37 \\
\hline & Total & 264 & 138 & 52 & 264 & 138 & 52 \\
\hline
\end{tabular}


were correct. Of these 182 identical predictions 168 $(92 \%)$ were correct, which was $64 \%$ of all 264 combinations. Regarding the six different activities we found high percentages of correct answers in upper body dressing and stair climbing (respectively 75\% and $80 \%$ of combinations), while a lower percentage was found in lower body dressing $(48 \%)$. In 82 combinations the team and the patients did not agree or were both uncertain. These categories of combinations were analyzed in Table 3.

In Table 3 all combinations of predictions of team and patient are presented related to the percentage of cases in which the patient actually performed the activities independently (percentages of success). Most independence was found when both the team and the patients expected this $(111 / 123=90 \%)$. When either the team or the patient answered 'yes' 17 of $40(43 \%)$ results were positive. In the category with other combinations (both 'uncertain' or 'no'/'uncertain') in eight of 42 activities $(19 \%)$ independence was achieved. When team and patients both did not expect independence only two of $59(3 \%)$ results were positive.

We compared self-care and mobility outcome of patients of four subgroups with different level and extent of lesion (Table 4). Regarding the self-care skills of patients with paraplegia both the team and patients expected independence of nearly all patients. All those patients achieved independence in eating and dressing the upper body. Lower body dressing was not always predicted correctly and only two of the 23 patients were not independent at discharge. Predictions of selfcare outcome of patients with tetraplegia varied strongly. About $40 \%$ of the activities were performed independently ( 25 of 63 ).

Regarding the mobility skills of patients with complete lesions the team and patients often agreed about the expected negative results of walking and stair climbing. Predictions of making car transfers showed a lot of variation in answers, while only one patient with complete tetraplegia and 13 with complete paraplegia achieved independence at discharge. Concerning functional walking of patients with complete paraplegia we found that seven out of 18 combinations were 'other combinations', which means that the team and patients did not agree or were both uncertain. The results at discharge of this group were poor.

If we focus on the mobility predictions of patients with incomplete lesions, we also found various combinations of predictions. If the team and patients both expected independence, the success percentage was high. One patient with incomplete tetraplegia became independent in walking and stair climbing, although both the team and the patient himself did not expect functional ambulation soon after admission.

Table 2 Combinations of identical predictions of the rehabilitation team and patients regarding independence in different activities of daily living with number and percentage of correct predictions $(n=44)$

\begin{tabular}{|c|c|c|c|c|c|c|c|c|c|c|c|}
\hline \multirow{2}{*}{$\begin{array}{l}\text { Activities of daily } \\
\text { living }\end{array}$} & \multirow{2}{*}{$\begin{array}{c}\text { Total of comb. } \\
\text { predictions } \\
n\end{array}$} & \multicolumn{2}{|c|}{ Both yes } & \multicolumn{4}{|c|}{ Both no } & \multicolumn{2}{|c|}{ Total both yes } & \multirow{2}{*}{$\begin{array}{c}\text { and no } \\
\%\end{array}$} & \multirow{2}{*}{$\begin{array}{l}\% \text { of } \\
\text { total }\end{array}$} \\
\hline & & $n$ & Corr. & $\%$ & $n$ & Corr. & $\%$ & $n$ & Corr. & & \\
\hline Eating & 44 & 28 & 25 & 89 & 1 & 1 & 100 & 29 & 26 & 90 & 59 \\
\hline Upper body dressing & 44 & 36 & 32 & 89 & 1 & 1 & 100 & 37 & 33 & 89 & 75 \\
\hline Lower body dressing & 44 & 21 & 19 & 90 & 2 & 2 & 100 & 23 & 21 & 91 & 48 \\
\hline Car transfer & 44 & 25 & 23 & 92 & 3 & 3 & 100 & 28 & 26 & 93 & 59 \\
\hline Walking & 44 & 7 & 6 & 86 & 22 & 21 & 95 & 29 & 27 & 93 & 61 \\
\hline Stair climbing & 44 & 6 & 6 & 100 & 30 & 29 & 97 & 36 & 35 & 97 & 80 \\
\hline Total & 264 & 123 & 111 & 90 & 59 & 57 & 97 & 182 & 168 & 92 & 64 \\
\hline Per cent of total & & $47 \%$ & & & $22 \%$ & & & $69 \%$ & & & \\
\hline
\end{tabular}

Table 3 Combinations of predictions of the rehabilitation team and patients regarding independence in different activities of daily living related to number and percentage of positive results in performing these activities independently at discharge $(n=44)$

\begin{tabular}{|c|c|c|c|c|c|c|c|c|c|c|c|c|c|}
\hline \multirow{2}{*}{$\begin{array}{l}\text { Activities of daily } \\
\text { living }\end{array}$} & \multirow{2}{*}{$\begin{array}{c}\text { Total of comb. } \\
\text { Predict. } \\
n\end{array}$} & \multicolumn{2}{|c|}{ Both yes } & \multirow[b]{2}{*}{$\%$} & \multicolumn{2}{|c|}{ One yes } & \multirow[b]{2}{*}{$\%$} & \multicolumn{3}{|c|}{ Other combinations } & \multicolumn{2}{|c|}{ Both no } & \multirow[b]{2}{*}{$\%$} \\
\hline & & $n$ & Pos & & $n$ & Result & & $n$ & Pos & $\%$ & $n$ & Pos & \\
\hline Eating & 44 & 28 & 25 & 89 & 10 & 4 & 40 & 5 & 1 & 20 & 1 & 0 & 0 \\
\hline Upper body dressing & 44 & 36 & 32 & 89 & 4 & 2 & 50 & 3 & 1 & 33 & 1 & 0 & 0 \\
\hline Lower body dressing & 44 & 21 & 19 & 90 & 12 & 6 & 50 & 9 & 2 & 22 & 2 & 0 & 0 \\
\hline Car transfer & 44 & 25 & 23 & 92 & 11 & 5 & 45 & 5 & 0 & 0 & 3 & 0 & 0 \\
\hline Walking & 44 & 7 & 6 & 86 & 1 & 0 & 0 & 14 & 4 & 29 & 22 & 1 & 5 \\
\hline Stair climbing & 44 & 6 & 6 & 100 & 2 & 0 & 0 & 6 & 0 & 0 & 30 & 1 & 3 \\
\hline Total & 264 & 123 & 111 & 90 & 40 & 17 & 43 & 42 & 8 & 19 & 59 & 2 & 3 \\
\hline
\end{tabular}


Table 4 Combinations of predictions of rehabilitation team and patients regarding independence of four subgroups with patients with different level and extent of lesion related to the percentage of positive results in performing different activities of daily living $(n=44)$

\begin{tabular}{|c|c|c|c|c|c|c|c|c|c|c|c|c|c|}
\hline \multirow[b]{2}{*}{ Subgroups } & \multirow[b]{2}{*}{ Predictions } & \multicolumn{2}{|c|}{ Eating } & \multicolumn{2}{|c|}{$\begin{array}{l}\text { Upper body } \\
\text { dressing }\end{array}$} & \multicolumn{2}{|c|}{$\begin{array}{l}\text { Lower body } \\
\text { dressing }\end{array}$} & \multirow{2}{*}{$\begin{array}{c}\text { Car } \\
n\end{array}$} & \multirow{2}{*}{$\begin{array}{c}\text { transfers } \\
\% \text { Pos }\end{array}$} & \multicolumn{2}{|c|}{ Walking } & \multicolumn{2}{|c|}{ Stair climbing } \\
\hline & & $n$ & $\%$ Pos & $n$ & $\%$ Pos & $n$ & $\%$ Pos & & & $n$ & $\%$ Pos & $n$ & $\%$ POS \\
\hline \multirow{4}{*}{$\begin{array}{l}\text { Complete } \\
\text { tetraplegia } \\
n=10\end{array}$} & Both yes & 3 & 33 & 5 & 80 & 0 & & 0 & & 0 & & 0 & \\
\hline & One yes & 2 & 0 & 3 & 33 & 2 & 50 & 4 & 25 & 0 & & 0 & \\
\hline & Other comb & 4 & 25 & 1 & 100 & 7 & 0 & 4 & 0 & 1 & 0 & 1 & 0 \\
\hline & Both no & 1 & 0 & 1 & 0 & 1 & 0 & 2 & 0 & 9 & 0 & 9 & 0 \\
\hline \multirow{4}{*}{$\begin{array}{l}\text { Incomplete } \\
\text { tetraplegia } \\
n=11\end{array}$} & Both yes & 3 & 67 & 9 & 67 & 4 & 75 & 7 & 100 & 5 & 100 & 5 & 100 \\
\hline & One yes & 7 & 43 & 0 & & 4 & 0 & 3 & 100 & 1 & 0 & 0 & \\
\hline & Other comb & 1 & 0 & 2 & 0 & 2 & 100 & 0 & & 3 & 67 & 3 & 0 \\
\hline & Both no & 0 & & 0 & & 1 & 0 & 1 & 0 & 2 & 50 & 3 & 33 \\
\hline \multirow{4}{*}{$\begin{array}{l}\text { Complete } \\
\text { paraplegia } \\
n=18\end{array}$} & Both yes & 17 & 100 & 17 & 100 & 14 & 95 & 15 & 87 & 1 & 0 & 0 & \\
\hline & One yes & 1 & 100 & 1 & 100 & 4 & 75 & 2 & 0 & 0 & & 1 & 0 \\
\hline & Other comb & 0 & & 0 & & 0 & & 1 & 0 & 7 & 0 & 2 & 0 \\
\hline & Both no & 0 & & 0 & & 0 & & 0 & & 10 & 0 & 15 & 0 \\
\hline \multirow{4}{*}{$\begin{array}{l}\text { Incomplete } \\
\text { paraplegia } \\
n=5\end{array}$} & Both yes & 5 & 100 & 5 & 100 & 3 & 100 & 4 & 75 & 1 & 100 & 1 & 100 \\
\hline & One yes & 0 & & 0 & & 2 & 100 & 1 & 100 & 0 & & 1 & 0 \\
\hline & Other comb & 0 & & 0 & & 0 & & 0 & & 3 & 67 & 0 & \\
\hline & Both no & 0 & & 0 & & 0 & & 0 & & 1 & 0 & 3 & 0 \\
\hline
\end{tabular}

Discrimination for age and degree of spasticity did not influence the results in any way.

\section{Discussion}

The importance of early prediction of neurological and functional outcome of spinal cord injury is frequently stressed. ${ }^{1,4,7}$ Prognosis of neurological recovery and functional abilities is useful in informing the patient and his family and making decisions about treatment. Forecasting length of stay, costs and equipment is not possible without this information. ${ }^{7}$

Prognosis of neurological recovery is based on neurological examinations in the acute phase. As patients are unconscious, uncooperative or in pain, this information is often not reliable. ${ }^{1}$ During the first days after the spinal cord injury both neurological improvement and regression can be seen, due to postinjury changes in the spinal cord or surgical and medical intervention. ${ }^{15}$ In an attempt to perform a more accurate assessment in the acute period, other methods are being studied such as motor and somatosensory evoked potentials. ${ }^{16}$

When patients have recovered from acute medical problems rehabilitation plans are made based on the prognosis of future functional abilities. Even when neurological recovery is small, especially of patients with complete lesions, the functional improvement in daily activity skills is usually substantial. ${ }^{6}$ Besides level and extent of lesion other factors play an important role in functional prognosis. ${ }^{4-6,8,10,11}$ Age, weight, comorbidity are prognostic indicators which are largely known from the start of rehabilitation. Influence of factors like spasticity, pain, motivation and psychosocial factors becomes clear in later phases. During inpatient rehabilitation maximal outcome is gained by intensive training of functional skills and multidisciplinary approach of problems. ${ }^{17}$ In order to use rehabilitation services efficiently rehabilitation programmes should be based on selected realistic goals.

Several studies focused on the functional outcome of patients with spinal cord injury. ${ }^{4-6}$ Predictions of the potential functional status are more reliable when they are founded on extensive multidisciplinary examination, including assessment of psychosocial circumstances. $^{5}$ Few studies actually dealt with the correlation between predictions and final outcome after rehabilitation. ${ }^{4}$ This is the first study in which the predictions of patients were also used.

All patients in this group followed a complete rehabilitation programme. Given the retrospective character of the study, both the professionals and the patients were not aware of scientific purposes at the time of treatment. It was not possible to treat patients blinded from the reported predictions, as confrontation with (discrepancy in) these expectations was part of the rehabilitation programme. However, patients were able to train all functional skills in consultation with the team, even if the team did not expect a positive result.

Unfortunately the data of 11 patients were not complete. At least part of this group recovered so quickly that discharge took place before assessment of predictions. The results of functional prognosis and outcome of the total group of 55 patients were probably better than our actual results.

In selecting the functional abilities we were dependent on the items in the RIS-DIS and assessment list of predictions. Questions concerning bladder and bowel care did not match with the functional data of skills concerning bladder voiding and defaecation. The six skills studied here were 
considered as the most essential skills in self-care and mobility at home and outdoors.

When we looked at the results of the professionals and the patients we found that the similarity in predicting outcome was remarkable. The team and the patients recorded about equal numbers of 'yes' and 'no' answers. The rehabilitation team related the first impression of the individual patient after some weeks of training to their experience in treating patients with spinal cord injury. Factors such as age, co-morbidity, spasticity and motivation were taken into account. If the opinions of the team were divided, 'uncertain' was noted. This might be an explanation for the higher number of 'uncertain' answers (19\%) of the team. The predictions of the patients were based on the progress that was made so far in the standard programme of training and the information about prognosis received from the professionals. We assume that their expectations were influenced by subjective factors like understanding and coping. When we compare the 73 negative predictions of the team and 76 of the patients with the 126 negative results, we have to conclude that both the team and the patients were inclined to be 'uncertain' instead of giving a negative answer, if independence was not expected. In our experience it can be useful to confront the patient with the disagreement in expectations, but it is important to know that the team did not predict outcome significantly better.

On average two-thirds of the cases (69\%), the patients and the team had unanimous predictions 'yes' or 'no', of which most $(92 \%)$ were correct. It appeared that the combination of predictions of both the team and the patients gave the most reliable prognosis. If they both predicted independence, $90 \%$ of the activities were performed independently compared to $85 \%$ respectively, $82 \%$ if we only used the separate expectations of the team and the patients. If they both did not expect independence only $3 \%$ of the final results were positive. This was not different from the percentage of correct separate negative predictions of the team and patients. Most agreement and the highest number of correct predictions were found for upper body dressing. In contradiction, results for lower body dressing were worse. In selecting goals for self-care it is important to be aware of these discrepancies.

Regarding the self-care outcome of patients with paraplegia we found a lot of agreement on the expected degree of independence of these patients. From former studies we know that most patients achieve full independence in daily activity skills. ${ }^{4,6}$ The high number of independence at discharge of these patients in this study was not surprising. It is justified to expect that patients with paraplegia can eat, dress and make transfers independently.

For patients with tetraplegia the functional recovery of the upper extremities determines the final degree of independence. A wide variation in expectations and functional results of self-care skills was found. It means that it was difficult to agree upon the prognosis of these patients, in spite of theoretical concepts. In this study age and degree of spasticity did not differentiate between good and false predictions of self-care outcome. The role of psychosocial factors and coping was not studied, but needs attention in further studies. We assume that those factors also play an important role in the prediction and results of final outcome. As long as early prediction of self-care outcome for patients with tetraplegia is difficult, gradual adjustment of selected goals has to be taken for granted.

In Beatrixoord Rehabilitation Centre much attention is paid to the independence in self-care activities and transfers. Walking with sophisticated devices is of second importance for patients with complete lesions during the inpatient rehabilitation period and only trained by patients with enough physical and mental indurance. The poor results regarding walking of patients with complete lesions are a logical consequence of this policy. Still, the professionals and patients seemed to hesitate in being pessimistic about future functional ambulation potential of patients with complete paraplegia. The expected and assessed results of patients with incomplete lesions (of all levels) varied widely. However, it seems clear that if the rehabilitation team and the patients agreed upon the expected independence in walking and stair climbing of patients with incomplete lesions, the results were mostly good. Again age and spasticity did not seem to play a role.

In conclusion, this study indicates the importance of assessing the expectations of both the rehabilitation team and the patient. The most reliable prediction of functional outcome after spinal cord injury was found when the expectations of both the professionals and the patients were combined. Soon after admission the prognosis of self-care was usually clear regarding patients with paraplegia, and of walking and stair climbing regarding patients with complete lesions. In this phase selection of specific goals seems reasonable. Early prediction of self-care outcome of patients with tetraplegia, and mobility outcome of patients with incomplete lesions, was more difficult. Gradual adjustment of rehabilitation objectives is needed during the course, in close collaboration between the individual patient and the team.

\section{References}

1 Waters RL, Adkins RH, Yakura JS, Sie I. Motor and sensory recovery following complete tetraplegia. Arch Phys Med Rehab 1993; 74: $242-247$.

2 Waters RL, Yakura YS, Adkins RH, Sie I. Recovery following complete paraplegia. Arch Phys Med Rehab 1992; 73: 784-789.

3 Waters RL, Adkins RH, Yakura JS, Sie I. Motor and sensory recovery following incomplete paraplegia. Arch Phys Med Rehab 1994; 75: $67-72$.

4 Woolsey RM. Rehabilitation outcome following spinal cord injury. Arch Neurol 1985; 42: 116-119.

5 Yarkony GM, et al. Benefits of rehabilitation for traumatic spinal cord injury. Arch Neurol 1987; 44: 93-96.

6 Schönherr MC, Groothoff JW, Mulder GA, Eisma WH. Functional outcome of patients with spinal cord injury. Clin Rehabil 1999; 13: $464-475$. 
7 Lazar LB, et al. Prediction of functional outcome by motor capability after spinal cord injury. Arch Phys Med Rehabil 1989; 70: $819-822$.

8 Mizukami M et al. Relationship between functional levels and movement in tetraplegic patients. A retrospective study. Paraplegia 1995; 33: $189-194$.

9 Welch RD, Lobley SJ, O'Sullivan SB, Freed MM. Functional independence in quadriplegia: critical levels. Arch Phys Med Rehabil 1986; 67: 235 - 240.

10 Yarkony GM, Roth EJ, Heinemann AW, Lovell L. Spinal cord injury rehabilitation outcome: the impact of age. J Clin Epidemiol 1988; 41: $173-177$.

11 DeVivo MJ et al. The influence of age at time of spinal cord injury on rehabilitation outcome. Arch Neurol 1990; 47: $687-$ 691.

12 Nene AV, Hermens HJ, Zilvold G. Paraplegic locomotion: a review. Spinal Cord 1996; 34: 507-523.
13 Schönherr MC, Groothoff JW, Mulder GA, Eisma WH Rehabilitation of patients with spinal cord lesions in the Netherlands: an epidemiological study. Spinal Cord 1996; 34: $679-683$.

14 ASIA/IMSOP. Standards for Neurological and Functional Classification of Spinal Cord Injury - Revised 1992. American Spinal Injury Association, Chicago, 1992.

15 Brown PJ, Marino RJ, Herbison GJ, Ditunno Jr JF. The 72-hour examination as a predictor of recovery in motor complete quadriplegia. Arch Phys Med Rehabil 1991; 72: 546-548.

16 Curt A, Dietz V. Electrophysiological recordings in patients with spinal cord injury: significance for predicting outcome. Spinal Cord 1999; 37: 157-165.

17 Yarkony GM et al. Functional skills after spinal cord injury rehabilitation: three-year longitudinal follow-up. Arch Phys Med Rehabil 1988; 69: $111-114$ 